\title{
On Common Property (E.A) for Hybrid Mappings in Gauge Spaces
}

\author{
Adnan Alhomaidan \\ Department of Mathematics, Faculty of Science, \\ King Abdulaziz University \\ P.O. Box 80081, Jeddah 21589, Saudi Arabia \\ alhomaidana@yahoo.com
}

\begin{abstract}
We discuss the common property (E.A) for hybrid pairs of single valued and multivalued mappings defined on gauge spaces in fixed point considerations.
\end{abstract}

\section{Introduction}

In 2002, Aamri and El Moutawakil ${ }^{[1]}$ introduced the property (E.A) in metric spaces as a generalization of the concept of noncompatible mappings and obtained some common coincidence point theorems for single-valued mappings satisfying a strict contractive condition. Subsequently, this property was extended to multivalued mappings independently by Kamran ${ }^{[2]}$ and Singh and Hashim ${ }^{[3]}$. Very recently, Liu, $\mathrm{Wu}$ and $\mathrm{Li}^{[4]}$ defined the common property (E.A) in metric spaces which contains the property (E.A) for a hybrid pair of single-valued and multivalued mappings. On the other hand, Frigon ${ }^{[5]}$ established the Banach contraction principle in complete gauge spaces. Frigon ${ }^{[6]}$ presented a fixed point theorem for multivalued contractions on complete gauge spaces which generalizes fixed point results of Nadler ${ }^{[7]}$ and Cain and Nashed ${ }^{[8]}$. The purpose of this paper to extend the common property (E.A) to gauge spaces and give some coincidence point results and common fixed point results for mappings satisfying this property.

\section{Preliminaries}

Let $\mathbf{E}=\left(\mathbf{E},\left\{d_{\alpha}\right\}_{\alpha \in \Lambda}\right)$ be a gauge space endowed with a gauge structure $\left\{d_{\alpha}\right.$ : $\alpha \in \Lambda\}$; here $\Lambda$ is a directed set (see Ref. [9, p. 198, 308, 414]). It is clear that a 
metric space is trivially a gauge space as the topology is given by one metric and a locally convex space, where the topology is given by a family of seminorms, is also a gauge space as each seminorm induces a pseudometric. For any $A, B \subset \mathbf{E}$, we define the generalized Hausdorff pseudometric induced by $d_{\alpha}$ to be

$$
D_{\alpha}(A, B)=\inf \left\{\varepsilon>0: \forall x \in A, y \in B, \exists x^{*} \in A, y^{*} \in B\right.
$$

such that $\left.d_{\alpha}\left(x, y^{*}\right)<\varepsilon, d_{\alpha}\left(x^{*}, y\right)<\varepsilon\right\}$ with the convention that $\inf (\phi)=\infty$. Let $\operatorname{dist}_{\alpha}(x, A)=\inf \left\{d_{\alpha}(x, y): y \in A\right\}$ for $A \subset \mathbf{E}$, and let $C D(\mathbf{E})$ denote the family of nonempty closed subsets of $\mathbf{E}$.

\section{Definition 1}

Let $f$ be a self-mapping of $\mathbf{E}$, and let $F$ be a mapping from $\mathbf{E}$ into $C D(\mathbf{E})$. A point $x \in \mathbf{E}$ is a coincidence point of $f$ and $F$ if $f x \in F x$. We denote the set of all coincidence points of $f$ and $F$ by $C(f, F)$.

\section{Definition 2}

Let $f$ be a self-mapping of $\mathbf{E}$, and let $F$ be a mapping from $\mathbf{E}$ into $C D(\mathbf{E})$. A point $x \in \mathbf{E}$ is a common fixed point of $f$ and $F$ if $x=f x \in F x$.

\section{Definition 3}

Let $F$ be a mapping from $\mathbf{E}$ into $C D(\mathbf{E})$. A self-mapping $f$ of $\mathbf{E}$ is said to be $F$-weakly commuting at $x \in \mathbf{E}$ if $f f x \in F f x$. (see Ref. [2]).

\section{Definition 4}

Let $f$ and $F$ be self-mappings of a gauge space E. We say that $(f, F)$ satisfies the property (E.A) if there exist a sequence $\left\{x_{n}\right\}$ in $\mathbf{E}$ and some $u \in \mathbf{E}$ such that

$$
\lim _{n \rightarrow \infty} d_{\alpha}\left(f x_{n}, u\right)=0=\lim _{n \rightarrow \infty} d_{\alpha}\left(F x_{n}, u\right)
$$

for all $\alpha \in \Lambda$. (see Ref. [10])

We follow Liu et al. ${ }^{[4]}$ and give the following:

\section{Definition 5}

Let $f, g$ be self-mappings of a gauge space $\mathbf{E}$, and let $F, G$ be mappings from $\mathbf{E}$ into $C D(\mathbf{E})$. We say that $(f, F)$ and $(g, G)$ satisfy the common property (E.A) if there exist sequences $\left\{x_{n}\right\},\left\{y_{n}\right\}$ in $\mathbf{E}$, some $u \in \mathbf{E}$, and $A, B$ in $C D(\mathbf{E})$ with $u A \cap B$ such that

$$
\lim _{n \rightarrow \infty} d_{\alpha}\left(f x_{n}, u\right)=0=\lim _{n \rightarrow \infty} d_{\alpha}\left(g y_{n}, u\right)
$$

and 


$$
\lim _{n \rightarrow \infty} D_{\alpha}\left(F x_{n}, A\right)=0=\lim _{n \rightarrow \infty} D_{\alpha}\left(G y_{n}, B\right)
$$

for all $\alpha \in \Lambda$.

\section{Results}

We first prove the following result which is a generalization of Theorem 2.3 ((a), (b)) of Liu et al. ${ }^{[4]}$.

\section{Theorem 6}

Let $\mathbf{E}=\left(\mathbf{E},\left\{d_{\alpha}\right\}_{\alpha \in \Lambda}\right)$ be a gauge space. Let $f, g$ be self-mappings of $\mathbf{E}$, and let $F, G$ be mappings from $\mathbf{E}$ into $C D(\mathbf{E})$ such that

(1) $(f, F)$ and $(g, G)$ satisfy the common property (E.A);

(2) for all $\alpha \in \Lambda, x, y \in \mathbf{E}$, and some $\left\{\lambda_{\alpha}\right\} \in[0,1[\Lambda$

$$
\begin{gathered}
D_{\alpha}(F x, G y) \leq \max \left\{d_{\alpha}(f x, g y), \lambda_{\alpha}\left[d i s t_{\alpha}(f x, F x)+d i s t_{\alpha}(g y, G y)\right],\right. \\
\left.\lambda_{\alpha}\left[d i s t_{\alpha}(f x, G y)+d i s t_{\alpha}(g y, F x)\right]\right\}
\end{gathered}
$$

(3) for every $x \in \mathbf{E}$ and every $\left.\left\{\varepsilon_{\alpha}\right\} \in\right] 0, \infty[\Lambda$, there exist $y \in F x$ and $z \in G x$ such that

$$
d_{\alpha}(f x, \mathrm{y}) \leq \operatorname{dist}_{\alpha}(f x, F x)+\varepsilon_{\alpha}
$$

and

$$
d_{\alpha}(g x, \mathrm{z}) \leq \operatorname{dist}_{\alpha}(g x, G x)+\varepsilon_{\alpha}
$$

for every $\alpha \in \Lambda$.

If $f(\mathbf{E})$ and $g(\mathbf{E})$ are closed, then

(a) $f$ and $F$ have a coincidence point;

(b) $g$ and $G$ have a coincidence point.

\section{Proof}

Since $(f, F)$ and $(g, G)$ satisfy the common property (E.A), there exist sequences $\left\{x_{n}\right\},\left\{y_{n}\right\}$ in $\mathbf{E}, u \in \mathbf{E}$, and $A, B \in C D(\mathbf{E})$ with $u \in A \cap B$ such that

$$
\begin{gathered}
\lim _{n \rightarrow \infty} D_{\alpha}\left(F x_{n}, A\right)=0=\lim _{n \rightarrow \infty} D_{\alpha}\left(G y_{n}, B\right) \\
\lim _{n \rightarrow \infty} d_{\alpha}\left(f x_{n}, u\right)=0=\lim _{n \rightarrow \infty} d_{\alpha}\left(g y_{n}, u\right)
\end{gathered}
$$

for all $\alpha \in \Lambda$.

Since $g(\mathbf{E})$ and $f(\mathbf{E})$ are closed, we have $u \in f(\mathbf{E}) \cap g(\mathbf{E})$, that is, there exists some $v, w \in \mathbf{E}$ such that $u=f v=g w$. 
Fix $\alpha \in \Lambda$. Using condition (2), we have

$$
\begin{gathered}
D_{\alpha}\left(F x_{n}, G w\right) \leq \max \left\{d_{\alpha}\left(f x_{n}, g w\right), \lambda_{\alpha}\left[\operatorname{dist}_{\alpha}\left(f x_{n}, F x_{n}\right)+\operatorname{dist}_{\alpha}(g w, G w)\right],\right. \\
\left.\lambda_{\alpha}\left[\operatorname{dist}_{\alpha}\left(f x_{n}, G w\right)+\operatorname{dist}_{\alpha}\left(g w, F x_{n}\right)\right]\right\} .
\end{gathered}
$$

Taking the limit as $n \rightarrow \infty$, we obtain

$$
\begin{aligned}
D_{\alpha}(A, G w) & \leq \max \left\{d_{\alpha}(u, g w), \lambda_{\alpha}\left[\operatorname{dist}_{\alpha}(u, A)+\operatorname{dist}_{\alpha}(g w, G w)\right],\right. \\
& \left.\lambda_{\alpha}\left[\operatorname{dist}_{\alpha}(u, G w)+\operatorname{dist}_{\alpha}(g w, A)\right]\right\} \\
& \leq \max \left\{0, \lambda_{\alpha}\left[0+\operatorname{dist}_{\alpha}(g w, G w)\right], \lambda_{\alpha}\left[\operatorname{dist}_{\alpha}(g w, G w)+0\right]\right\} \\
& =\lambda_{\alpha} \operatorname{dist}_{\alpha}(g w, G w) .
\end{aligned}
$$

Suppose that $\operatorname{dist}_{\alpha 0}(g w, G w) \neq 0$ for some $\alpha_{0} \in \Lambda$. Then, since $g w \in A$, by the definition of $D_{\alpha}$, we have

$$
\begin{aligned}
\operatorname{dist}_{\alpha 0}(g w, G w) & \leq D_{\alpha 0}(A, G w) \\
& \leq \lambda_{\alpha 0} \operatorname{dist}_{\alpha 0}(g w, G w),
\end{aligned}
$$

which is a contradiction since $0 \leq \lambda_{\alpha 0}<1$. Thus $\operatorname{dist}_{\alpha}(g w, G w)=0$. Since this holds for all $\alpha$ in $\Lambda$, it follows from condition (3) that $g w \in G w$. Similarly, by setting $x=v$ and $y=y_{n}$ in inequality (1) and taking the limit as $n \rightarrow \infty$, we get $f v \in F v$.

If we set $g=f$ and $G=F$ in Theorem 6, we immediately get the following corollary, which improves and generalizes Theorem 1 of Aamri and El Moutawakil $^{[1]}$ and Theorem 3.3 of Kamran ${ }^{[2]}$.

\section{Corollary 7}

Let $\mathbf{E}=\left(\mathbf{E},\left\{d_{\alpha}\right\}_{\alpha \in \Lambda}\right)$ be a gauge space. Let $f$ be a self-mappings of $E$, and let $F$ be a mapping from $\mathbf{E}$ into $C D(\mathbf{E})$ such that

(1) $(f, F)$ satisfies the common property (E.A);

(2) for all $\alpha \in \Lambda, x, y \in \mathbf{E}$, and some $\left\{\lambda_{\alpha}\right\} \in\left[0,1\left[{ }^{\Lambda}\right.\right.$,

$$
\begin{gathered}
D_{\alpha}(F x, F y) \leq \max \left\{d_{\alpha}(f x, f y), \lambda_{\alpha}\left[\operatorname{dist}_{\alpha}(f x, F x)+\operatorname{dist}_{\alpha}(f y, F y)\right],\right. \\
\left.\lambda_{\alpha}\left[\operatorname{dist}_{\alpha}(f x, F y)+\operatorname{dist}_{\alpha}(f y, F x)\right]\right\} ;
\end{gathered}
$$

(3) for every $x \in \mathbf{E}$ and every $\left.\left\{\varepsilon_{\alpha}\right\} \in\right] 0, \infty\left[{ }^{\Lambda}\right.$, there exist $y \in F x$ such that

$$
d_{\alpha}(f x, y) \leq \operatorname{dist}_{\alpha}(f x, F x)+\varepsilon_{\alpha}
$$

for every $\alpha \in \Lambda$.

If $f(\mathbf{E})$ is closed, then $f$ and $F$ have a coincidence point.

The following result generalizes Theorem 2.3(c)-(e) of Liu, Wu and $\mathrm{Li}^{[4]}$. 


\section{Theorem 8}

Let $\mathbf{E}, f, g, F$ and $G$ be as in Theorem 6 and satisfy (1), (2) and (3). Then we have the following:

(a) If $f$ is $F$-weakly commuting at $v$ and $f f v=f v$ for some $v \in C(f, F)$, then $f$ and $F$ have a common fixed point.

(b) If $g$ is $G$-weakly commuting at $w$ and $g g w=g w$ for some $w \in C(g, G)$, then $g$ and $G$ have a common fixed point.

(c) If (a) and (b) hold, then $f, g, F$ and $G$ have a common fixed point.

\section{Proof}

As in Theorem 6, there exists $v, w$ in $\mathbf{E}$ such that $f v \in F v, g w \in G w$ and $u=$ $f v=g w$.

(a) Since $f$ is $F$-weakly commuting at $v$ and $f f v=f v$, it follows that $f v=f$ $f v \in F f v$. Consequently, we have $u=f u \in F u$. This shows that $u$ is a common fixed point of $f$ and $F$.

(b) Similar to (a).

(c) Follows from (a) and (b).

The following corollary generalizes and improves Theorem 3.10 of Kamran ${ }^{[2]}$.

\section{Corollary 9}

Let $\mathbf{E}, f$ and $F$ be as in Theorem 6 and satisfy (1), (2) and (3). If $f$ is $F$-weakly commuting at $v$ and $f f v=f v$ for some $v \in C(f, F)$, then $f$ and $F$ have a common fixed point.

\section{Theorem 10}

Let $\mathbf{E}=\left(\mathbf{E},\left\{d_{\alpha}\right\}_{\alpha \in \Lambda}\right)$ be a gauge space. Let $f, g$ be self-mappings of $\mathbf{E}$, and let $F, G$ be mappings from $\mathbf{E}$ into $C D(\mathbf{E})$ such that

(1) $(f, F)$ and $(g, G)$ satisfy the common property (E.A);

(2) for all $\alpha \in \Lambda$, there exists a continuous nondecreasing function $\varphi_{\alpha}: \mathbb{R}^{+}$ $\rightarrow \mathbb{R}^{+}$with $0<\varphi_{\alpha}(t)<t$ for $t>0$ such that for all $x, y \in \mathbf{E}$, we have

$$
\begin{gathered}
D_{\alpha}(F x, G y) \leq \varphi_{\alpha}\left(\operatorname { m a x } \left\{d_{\alpha}(f x, g y), \operatorname{dist}_{\alpha}(f x, F x), \operatorname{dist}_{\alpha}(g y, G y),\right.\right. \\
\left.\left.\operatorname{dist}_{\alpha}(f x, G y), \operatorname{dist}_{\alpha}(g y, F x)\right\}\right) ;
\end{gathered}
$$

(3) for every $x \in \mathbf{E}$ and every $\left.\left\{\varepsilon_{\alpha}\right\} \in\right] 0, \infty\left[{ }^{\Lambda}\right.$, there exist $y \in F x$ and $z \in G x$ such that

$$
d_{\alpha}(f x, y) \leq \operatorname{dist}_{\alpha}(f x, F x)+\varepsilon_{\alpha}
$$

and

$$
d_{\alpha}(g x, z) \leq d i s t_{\alpha}(g x, G x)+\varepsilon_{\alpha}
$$


for every $\alpha \in \Lambda$.

If $f(\mathbf{E})$ and $g(\mathbf{E})$ are closed, then

(a) $f$ and $F$ have a coincidence point;

(b) $g$ and $G$ have a coincidence point.

\section{Proof}

Since $(f, F)$ and $(g, G)$ satisfy the common property (E.A), there exist sequences $\left\{x_{n}\right\},\left\{y_{n}\right\}$ in $\mathbf{E}, u \in \mathbf{E}$, and $A, B \in C D(\mathbf{E})$ with $u \in A \cap B$ such that

$$
\begin{gathered}
\lim _{n \rightarrow \infty} D_{\alpha}\left(F x_{n}, A\right)=0=\lim _{n \rightarrow \infty} D_{\alpha}\left(G y_{n}, B\right) \\
\lim _{n \rightarrow \infty} d_{\alpha}\left(f x_{n}, u\right)=0=\lim _{n \rightarrow \infty} d_{\alpha}\left(g y_{n}, u\right)
\end{gathered}
$$

for all $\alpha \in \Lambda$.

Since $g(\mathbf{E})$ and $f(\mathbf{E})$ are closed, as before, there exists some $v, w \in \mathbf{E}$ such that $u=f v=g w$.

Fix $\alpha \in \Lambda$. Using condition (2), we have

$$
\begin{gathered}
D_{\alpha}\left(F x_{n}, G w\right) \leq \varphi_{\alpha}\left(\operatorname { m a x } \left\{d_{\alpha}\left(f x_{n}, g w\right), \operatorname{dist}_{\alpha}\left(f x_{n}, F x_{n}\right), \operatorname{dist}_{\alpha}(g w, G w),\right.\right. \\
\left.\left.\operatorname{dist}_{\alpha}\left(f x_{n}, G w\right), \operatorname{dist}_{\alpha}\left(g w, F w_{n}\right)\right\}\right) .
\end{gathered}
$$

Taking the limit as $n \rightarrow \infty$, we obtain

$$
\begin{aligned}
D_{\alpha}(A, G w) & \leq \varphi_{\alpha}\left(\operatorname { m a x } \left\{d_{\alpha}(u, g w), \operatorname{dist}_{\alpha}(u, A), \operatorname{dist}_{\alpha}(g w, G w),\right.\right. \\
& \left.\left.\operatorname{dist}_{\alpha}(u, G w), \operatorname{dist}_{\alpha}(g w, A)\right\}\right) \\
& \leq \varphi_{\alpha}\left(\operatorname{dist}_{\alpha}(g w, G w)\right) .
\end{aligned}
$$

Suppose that dist $t_{\alpha 0}(g w, G w) \neq 0$ for some $\alpha_{0} \in \Lambda$. Then, since $g w \in A$, by the definition of $D_{\alpha}$, we have

$$
\begin{aligned}
\operatorname{dist}_{\alpha 0}(g w, G w) & \leq D_{\alpha 0}(A, G w) \\
& \leq \varphi_{\alpha 0}\left(\operatorname{dist}_{\alpha 0}(g w, G w)\right) \\
& <\operatorname{dist}_{\alpha 0}(g w, G w) .
\end{aligned}
$$

This is a contradiction. Thus dist $(g w, G w)=0$, and since this holds for all $\alpha$ in $\Lambda$, it follows from condition (3) that $g w \in G w$.

Similarly, by setting $x=v$ and $y=y_{n}$ in inequality (3) and taking the limit as $n \rightarrow \infty$, we get $f v \in F v$.

\section{Theorem 11}

Let $\mathbf{E}, f, g, F$ and $G$ be as in Theorem 10 and satisfy (1), (2) and (3). Then we have the following: 
(a) If $f$ is $F$-weakly commuting at $v$ and $f f v=f v$ for some $v \in C(f, F)$, then $f$ and $F$ have a common fixed point.

(b) If $g$ is $G$-weakly commuting at $w$ and $g g w=g w$ for some $w \in C(g, G)$, then $g$ and $G$ have a common fixed point.

(c) If (a) and (b) hold, then $f, g, F$ and $G$ have a common fixed point.

Theorems 10 and 11 generalize Theorem 2.10 of Liu, et al. ${ }^{[4]}$.

The notion of single-valued contraction (in the usual sense) has been extended by some authors. An important extension was given by Cain and Nashed ${ }^{[3]}$ in Hausdorff locally convex spaces $\left(\mathbf{E},\left\{|\cdot|_{\alpha}\right\}_{\alpha \in \Lambda}\right)$, where $\left\{|\cdot|_{\alpha}\right\}_{\alpha \in \Lambda}$ is a family of seminorms. But in most of those extension, the contraction $F$ satisfies the following restrictive condition:

$$
\text { if }|x-y|_{\alpha}=0 \text {, then }|F x-F y|_{\alpha}=0 .
$$

For example, consider $\mathbf{E}=\prod_{n \in \mathbf{N}} X_{n}$ with $\left(X_{n},|| n.\right)$ Banach spaces and $F=\left(F_{1}\right.$, $\left.F_{2}, \ldots\right)$ is such that there exists a matrix $A=\left(a_{i j}\right)$ with nonnegative entries such that for every $n \in \mathbf{N}$,

$$
\left|F_{n} x-F_{n} y\right|_{n} \leq a_{n 1}\left|x_{1}-y_{1}\right|_{1}+a_{n 2}\left|x_{2}-y_{2}\right|_{2}+\ldots
$$

If $F$ is a contraction, one can choose the matrix $A$ lower triangular. So $F_{n}$ does not depend on $x_{n+1}, x_{n+2}, \ldots$. In view of the following definition, we allow $a_{i j}>$ 0 for every $i, j \in \mathbf{N}$ in such situations. For details, see Ref. [5].

\section{Definition 12}

Let $f$ be a self-mapping of $\mathbf{E}$, and let $F$ be a mapping from $\mathbf{E}$ into $C D(\mathbf{E})$ such that $F(\mathbf{E}) \subset f(\mathbf{E})$. Then $F$ is called an admissible $f$-contraction (cf. Ref. [6]) with $\left\{k_{\alpha}\right\} \in[0,1[\Lambda$ if

(i) for every $\alpha \in \Lambda$,

$$
D_{\alpha}(F x, F y) \leq k_{\alpha} d_{\alpha}(f x, f y)
$$

for every $x, y \in \mathbf{E}$;

(ii) for every $x \in \mathbf{E}$ and every $\left.\left\{\varepsilon_{\alpha}\right\} \in\right] 0, \infty\left[{ }^{\Lambda}\right.$, there exists $y \in F x$ such that

$$
D_{\alpha}(x, y) \leq \operatorname{dist}_{\alpha}(x, F x)+\varepsilon_{\alpha}
$$

for every $\alpha \in \Lambda$.

Notice that if $\Lambda=\mathbf{N}$, then a multivalued mapping $F$ can be an $f$-contraction in the sense of Definition 12 without being an $f$-contraction in the usual sense when $\mathbf{E}$ is equipped with the metric

$$
d(x, y)=\sum_{n \in \mathbf{N}} \frac{d_{n}(x, y)}{2^{n}\left(1+d_{n}(x, y)\right) .}
$$


As an application of our result, we derive the following extension of the main result of Frigon ${ }^{[6]}$, which itself is a generalization of Nadler's contraction principle $^{[7]}$ and Cain and Nashed's result [Ref. 8, Theorem 2.2]. It is worth noting that in the case when $F$ is single-valued, unlike other authors, we do not require the following restrictive condition:

$$
\text { if }|x-y|_{\alpha}=0 \text {, then }|F x-F y|_{\alpha}=0 .
$$

\section{Theorem 13}

Let $f$ be a self-mapping of a complete gauge space $\mathbf{E}$, and let $F$ be a mapping from $\mathbf{E}$ into $C D(\mathbf{E})$ such that $F(\mathbf{E}) \subset f(\mathbf{E})$. If $F$ is an admissible $f$-contraction and $f(\mathbf{E})$ is closed, then $f$ and $F$ have a coincidence point.

\section{Proof}

Since $F(\mathbf{E}) \subset f(\mathbf{E})$ so for any given $x_{0} \in \mathbf{E}$, there is an $x_{1} \in \mathbf{E}$ such that $y_{1}=$ $f x_{1} \in F x_{0}$. This is possible since $F(\mathbf{E}) \subset f(\mathbf{E})$. Fix $\alpha \in \Lambda$. We can find $y_{2}=f x_{2}$ $\in F x_{1}$ such that

$$
\begin{gathered}
d_{\alpha}\left(y_{1}, y_{2}\right) \leq \operatorname{dist}_{\alpha}\left(y_{1}, F x_{1}\right)+k_{\alpha} \\
\leq D_{\alpha}\left(F x_{0}, F x_{1}\right)+k_{\alpha} .
\end{gathered}
$$

Continuing in this way, we obtain a sequence $\left\{y_{n}\right\}$ in $\mathbf{E}$ with $y_{n+1}=f x_{n+1} \in F x_{n}$ such that

$$
d_{\alpha}\left(y_{n}, y_{n+1}\right) \leq D_{\alpha}\left(F x_{n-1}, F x_{n}\right)+k_{\alpha}^{n}, \quad n=1,2, \ldots
$$

It is easy to show that $\left\{y_{n}\right\}$ is Cauchy with respect to $d_{\alpha}$. Since we can do this for any $\alpha \in \Lambda$ and the sequence $\left\{y_{n}\right\}$ is Cauchy, there is $u \in \mathbf{E}$ such that

$$
\lim _{n \rightarrow \infty} d_{\alpha}\left(y_{n}, u\right)=\lim _{n \rightarrow \infty} d_{\alpha}\left(f x_{n}, u\right)=0
$$

for all $\alpha \in \Lambda$. Notice that $u \in A$ and

$$
\lim _{n \rightarrow \infty} D_{\alpha}\left(F x_{n}, A\right)=0
$$

for all $\alpha \in \Lambda$ and some $A \in C D(\mathbf{E})$. As a result, $(f, F)$ satisfies the common property $(E . A)$ and so the result follows from Theorem 6 .

\section{Acknowledgement}

The author thanks the referee for his valuable suggestions towards the improvement of the paper. 


\section{References}

[1] Aamri, M. and El-Moutawakil, D., Some new common fixed point theorems under strict contractive conditions, J. Math. Anal. Appl., 270: 181-188 (2002).

[2] Kamran, T., Coincidence and fixed points for hybrid strict contractions, J. Math. Anal. Appl., 299: 235-241 (2004).

[3] Singh, S.L. and Hashim, A.M., New coincidence and fixed point theorems for strictly contractive hybrid maps, Austral. J. Math. Anal. Appl., 2: 1-7 (2005).

[4] Liu, Y., Wu, J. and Li, Z., Common fixed points of single-valued and multivalued maps, Internat. J. Math. Sci., 19: 3045-3055 (2005).

[5] Frigon, M., Fixed point results for generalized contractions in gauge spaces and applications, Proc. Amer. Math. Soc., 128: 2957-2965 (2000).

[6] Frigon, M., Fixed point results for multivalued contraction on gauge spaces, Ser. Math. Anal. Appl., 4: 175-181 (2002).

[7] Nadler, Jr S.B., Multivalued contraction mappings, Pacific J. Math., 30: 415-487 (1969).

[8] Cain, G.L. and Nashed, M.Z., Fixed points and stability for a sum of two operators in locally convex spaces, Pacific J. Math., 39: 581-592 (1971).

[9] Dugundji, J., Topology, Ally and Bacon, Boston (1966).

[10] Alhomaidan, A., On property (E.A) in gauge spaces, Internat. J. Math. Game Theory and Algebra, 15 (1): 1-15 (2006). 


\title{
بعض النتائج عن خاصية (E.A) لبعض العلاقات المزدوجة على فراغ قيــج
}

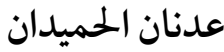 \\ قسم الرياضيات ، كلية العلوم ، جامعة الملك عبدالعزيلة العزيز \\ جســة - المملكة العربية السعودية \\ alhomaidana@yahoo.com
}

المستخلص. يناقش هذا البحث خاصية (E.A) للعلاقات الثنائية ذات

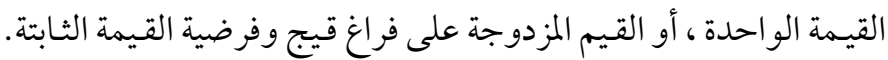

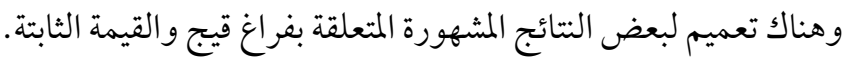

